\title{
HISTORY, GEOMETRY, STRUCTURE: INTERDISCIPLINARY ANALYSIS OF A HISTORICAL BRIDGE
}

\author{
N. Bruno ${ }^{1}$, E. Coïsson ${ }^{1}$, F. Diotri ${ }^{2}$, L. Ferrari ${ }^{1}$, S. Mikolajewska ${ }^{1}$, U. Morra di Cella ${ }^{2}$, R. Roncella ${ }^{1}$, A. Zerbi ${ }^{1}$ \\ ${ }^{1}$ Department of Engineering and Architecture, University of Parma, Italy - (nazarena.bruno, eva.coisson, lia.ferrari, \\ sandra.mikolajewska, riccardo.roncella, andrea.zerbi)@unipr.it \\ ${ }^{2}$ Agenzia Regionale Protezione Ambiente Valle d'Aosta (ARPA) - (f.diotri, u.morradicella)@arpa.vda.it
}

\section{Commission VI, WG VI/4}

KEY WORDS: historical monitoring, geometrical proportions, TLS survey, photogrammetry, historical bridge

\begin{abstract}
:
In this paper, an integrated analysis of the geometric and photogrammetric surveys on a $19^{\text {th }}$ century bridge is proposed as an instrument for the comprehension of the historical evolution of its structural disorders, of its present conservation status and as a reliable base to define future interventions. The studied 20 arches brick masonry bridge in Northern Italy, that reaches the length of nearly 600 meters, constitutes a very interesting case study, not only for the technical and structural issues related to its restoration and use (with increased traffic loads) but also for its geometry, that has strict connections with the design methods that lead its construction, based on proportions, and whose changes in time embody the present symptoms of possible structural illnesses. Moreover, the bridge shows many decay phenomena, whose treatment will require a large part of the restoration resources. For this reason, a precise quantification of the affected surfaces, also in the curved parts, is important for the definition of conservative interventions. To this aim, possible methods of mapping and measuring the decay phenomena starting from the photogrammetric survey are also discussed.
\end{abstract}

\section{INTRODUCTION}

The $19^{\text {th }}$ century "Ponte Taro" bridge, close to Parma in Northern Italy, is a very important monument, both from a cultural and strategic point of view. Its design was carried out following the historical 'proportional theory': empiricism, indeed, has been at the base of the main architectural constructions at least until the $17^{\text {th }}$ Century, but this geometrical method of structural design has been used, in particular for bridges, until the beginning of the 19th Century, testifying the permanence of empiric calculation for masonry, despite the availability of the new theories of the newborn Building Science (Benvenuto, 1981). Given the proportional and empiric approach of its designer, there is therefore a strict connection between the bridge geometry and its structural features. Thus the present paper aims at inspecting these connections, starting from a thorough (and complex, due to the large dimensions) geometric and photogrammetric survey and analysing its results in terms of deformations, structural disorders and decay phenomena, thanks to an interdisciplinary team and approach.

\section{THE CASE STUDY: THE "MARIA LUIGIA" $19^{\mathrm{TH}}$ CENTURY BRIDGE IN PARMA}

The Roman bridge built to let the via Emilia pass over the Taro River progressively deteriorated and at last collapsed in 1276. Since then, crossing the river in this point was possible only with boats (Basteri and Dall'Acqua, 2004). Finally, Maria Luigia, wife of Napoleon I and duchess of Parma, commissioned the construction of a new bridge to engineer Antonio Cocconcelli and the construction took place between 1816 and 1821. After several calculations about the foreseen floods, reported in (Cocconcelli, 1825), the choice was made for a series of 17 three-centered, depressed arches, with a $24 \mathrm{~m}$ span and a $6.6 \mathrm{~m}$ rise, resting on rectangular pillars with rounded ends to reduce the water impact, whose foundations were underpinned with a layer of $5 \mathrm{~m}$ long timber poles. Externally, all the structure is made of brick masonry, but internally also river cobblestones mixed with mortar were used. Unfortunately, during the construction, in 1818 floods devastated the building yard and Cocconcelli was forced to change his project, adding three arches, deepening the foundations and inserting oculi above the pillars to ease the water outflow (Cocconcelli, 1825). The bridge thus reached 20 arches and $565.5 \mathrm{~m}$ in length.

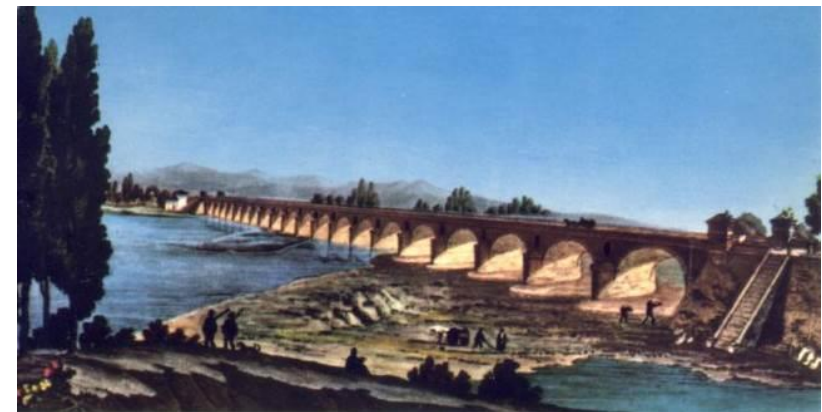

Figure 1. A historical view of the Ponte Taro bridge.

During the Second World War the bridge was hit by several bombings, whose signs are still visible on the masonry surface. In time, also some car accidents occurred, with vehicles braking through the parapet, and interventions were made both on the foundations and on the elevation (Basteri and Dall'Acqua, 2004). Nowadays the bridge is vehicle accessible and also trucks, directed to a nearby concrete factory, pass on it, despite its limited width $(8 \mathrm{~m})$ : it is clearly becoming more and more inadequate to modern traffic and it threatens the safety of pedestrian and bicycle riders. Moreover, several fragments of bricks have detached from the intrados of the arches, posing a 
danger to the underlying activities. The time has come for a thorough restoration project and the Municipalities involved (Parma, Ponte Taro and Noceto) have commissioned studies to inspect history, geometry and structure of this ancient infrastructure as a base for a future intervention.

\section{THE GEOMETRICAL AND PHOTOGRAMMETRIC SURVEY}

The surveyed bridge belongs to a national road that crosses

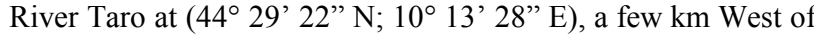
the city of Parma (Italy). Including the ramps, the bridge is about $850 \mathrm{~m}$ long and about $9 \mathrm{~m}$ wide. The road plane on bridge deck is about $10.5 \mathrm{~m}$ higher than the riverbed, a large floodplain with deposits of silt, sands and gravel and only a small stream channel with water at survey epoch (September 2018).

The peculiar shape and dimension of the bridge (about 600 meters in length, not considering the ramps, height between 10 and 13 meters, width 9 meters) required a careful planning of all the surveying procedures, also taking into account street-level traffic conditions, accessibility of the riverbed and so on. To limit possible interferences, to ensure that the operators can perform the survey in a safe environment and still to be able of acquiring relevant, detailed information of the upper part of the bridge (asphalt condition, parapets, etc.), an integrated survey using proximity range UAV photogrammetry, laser scanning and terrestrial photogrammetry with DSLR high resolution digital cameras has been considered the most appropriate approach. National Road authorities authorized, for a short period of time, the total closure of the road, to allow performing ground operations in total safety. Being the road a strategic major traffic artery at national scale such operations had to be performed in the shortest period of time possible.

In order to provide a stable control of the survey that could efficiently register all the different surveyed data in a common reference system, more than 100 targets have been placed both at the surface of the bridge and on the ground areas surrounding the bridge. All the points have been surveyed using both total station (Topcon IS200) and GNSS (Geomax Zenith20) measurement to improve reliability. This was followed by the terrestrial laser scanner (TLS) and photogrammetric survey, both from the ground level and, as far as photogrammetric survey is concerned, using also a fixed and a rotary-wing UAV.

More than 50 TLS scans (Leica Scanstation C-10) were carried out which allowed to obtain point clouds (Figure 2) with a Ground Sampling Distance (GSD) of about one centimeter. However, due to the strict time limits imposed by the road closure, just 5 scans have been performed on the upper part (road) of the bridge. The other 45 TLS scans were acquired from the riverbed. Specifically, in order to reconstruct bridge sides, arches, etc., for each arc two scans on opposite sides were made, connected to each other in a closed traverse. The resolution, quite high if the actual representation scale requested is considered, is of the same order of magnitude of the one planned for UAV and terrestrial photogrammetric survey $(0.7 \div 1.0 \mathrm{~cm})$, and allows a reconstruction of fine details if, during the restoration analysis, some local elements would require a more in depth analysis.

The terrestrial images (ca. 650 images) were obtained using wide-angle optics $(18 \div 35 \mathrm{~mm})$ in order to avoid occlusions due to the relevant presence of vegetation around the area of the bridge with a Nikon D3X, implementing two pseudo-nadiral strips on both sides of the structure. The images acquired in the pseudo-nadiral strips were integrated with other convergent images to derive geometry and state of decay of the intrados of every single arch.

For the UAV acquisition (Figure 3) two different platforms were used: for framing the entire region of the bridge and its surrounding (besides the river, also the industrial and residential neighborhood were documented) a fixed-wing eBee with RTK differential positioning functionality were considered optimal. The eBee RTK is equipped with a $20 \mathrm{Mp}$ compact S.O.D.A. camera with $10.6 \mathrm{~mm}$ focal length ( $35 \mathrm{~mm}$ equivalent: $29 \mathrm{~mm}$ ). The RGB camera (resolution $5472 \times 3648$ pixel, pixel size 2.4 $\mu \mathrm{m})$ acquires nadiral images with exposure parameters set automatically. The on-board receiver can process L1 and L2 GPS and GLONASS data and receive the differential corrections from the master station or from the control centre of a Continuously Operating Reference Station network via the flight control software and a ground radio modem. Camera position are stored as geo-tags in the image metadata and in the flight log. A Geomax Zenith20, set on a benchmark at the Eastern bridge end, has been used as master station.

Two flights have been executed with the eBee, about half an hour apart from each other. The former is made of 11 strips, flown along the bridge direction, with forward and side overlap of about $50 \%$ and $70 \%$ respectively (covered area: about $740 \mathrm{~m}$ x $370 \mathrm{~m}$ ). The latter consists of 7 strips, flown across the bridge just East of bridge centre, almost at the same height as the previous flight and with roughly the same overlaps (covered area: about $370 \mathrm{~m} \times 220 \mathrm{~m}$ ). The flight altitude have been quite low $(70 \div 75 \mathrm{~m})$ due to limitation imposed by the presence of nearby airports facilities. The two eBee flights have been combined in a single block and processed using Agisoft photoscan (version 1.4.3). The high resolution bridge survey flight, on the contrary, was performed using a rotary-wing DJI Phantom 4 UAV. The Dji Phantom4 is equipped with a $20 \mathrm{Mp}$ CMOS sensor with $8.8 \mathrm{~mm}$ focal length ( $35 \mathrm{~mm}$ equivalent: 24 $\mathrm{mm}$ ). The FC6310 RGB camera (resolution $5472 \times 3078$ pixel, pixel size $2.5 \mu \mathrm{m})$ is mounted on a stabilized gimbal with controllable pitch range from $-90^{\circ}$ to $+30^{\circ}$. The single frequency on-board GNSS receiver is fit for navigation purposes, not for accurate georeferencing. In this case, one nadiral strip (flight altitude ca. $50 \mathrm{~m}$ ) and two lateral oblique strip (at ca. $30 \mathrm{~m}$ from the bridge) were performed acquiring more than 610 images. A first high resolution acquisition of the upper part of the bridge was performed with a single image strip, flown manually with the Phantom4, with an average $80 \%$ forward overlap at ground level. While still under manual control and without landing, a second single strip has been executed, at a higher flying height but with a slightly larger forward overlap (as a matter of fact, however, the overlap is far from constant in both strips). The second, auxiliary, strip was performed with the aim of providing a better tying geometry between the higher altitude eBee acquisition and the DJI's. Finally, two oblique, straight strips documenting the sides of the bride and providing a connection between the riverbed, terrestrial DSLR images and the nadiral UAV-based acquisitions, were performed.

The GNSS positions of the targets as well as of the higher UAV flight camera stations, determined in the national reference frame ETRF2000(2008), have been converted to a local Cartesian reference system centred at mid bridge, with $\mathrm{z}$ origin on the reference ellipsoid, $\mathrm{z}$ axis along the ellipsoid normal and 
y axis parallel to the North axis of the UTM $32 \mathrm{~N}$ fuse of the ETRS89 datum.

The point clouds obtained by the three surveys (TLS, terrestrial photogrammetry and UAV) were than registered in the same reference system and compared using the Ground Control Points acquired, highlighting a discrepancy of ca. $17 \mathrm{~mm}$. After a careful manual inspection of the differently acquired point clouds and the removal of artefacts, gross errors, outliers etc., the two (TLS and image-based) point clouds were merged together and triangulated, allowing to obtain a final tridimensional mesh of the entire bridge and its surroundings.

As far as drawing and survey restitution operations are concerned, the mesh obtained was subjected to section operations with different vertical and horizontal planes. Through this operation it was possible to extrapolate all the data necessary for the elaboration of the traditional geometric survey documents
Two elevations of the bridge, a dimensioned plane, a longitudinal section, and four transversal sections in correspondence with the most significant arches and piers were then created (the first, the last, and two intermediate ones). All the drawings were produced with a higher precision than the traditional representation used for structures characterized by extremely significant dimensions, such as this one (it was used a scale of 1:50). As the point cloud obtained can be considered a sort of open database, it is possible to carry out further detailed sections at any time.

The $3 \mathrm{~d}$ reconstructed mesh was also imported back in Agisoft Photoscan, along with all the acquired (and oriented) UAV and DSLR images to produce orthophotos of the bridge elevations (sides) and intrados of its vaults. Such product was particularly helpful for the subsequent surface deterioration analysis as will be described in the next sections.

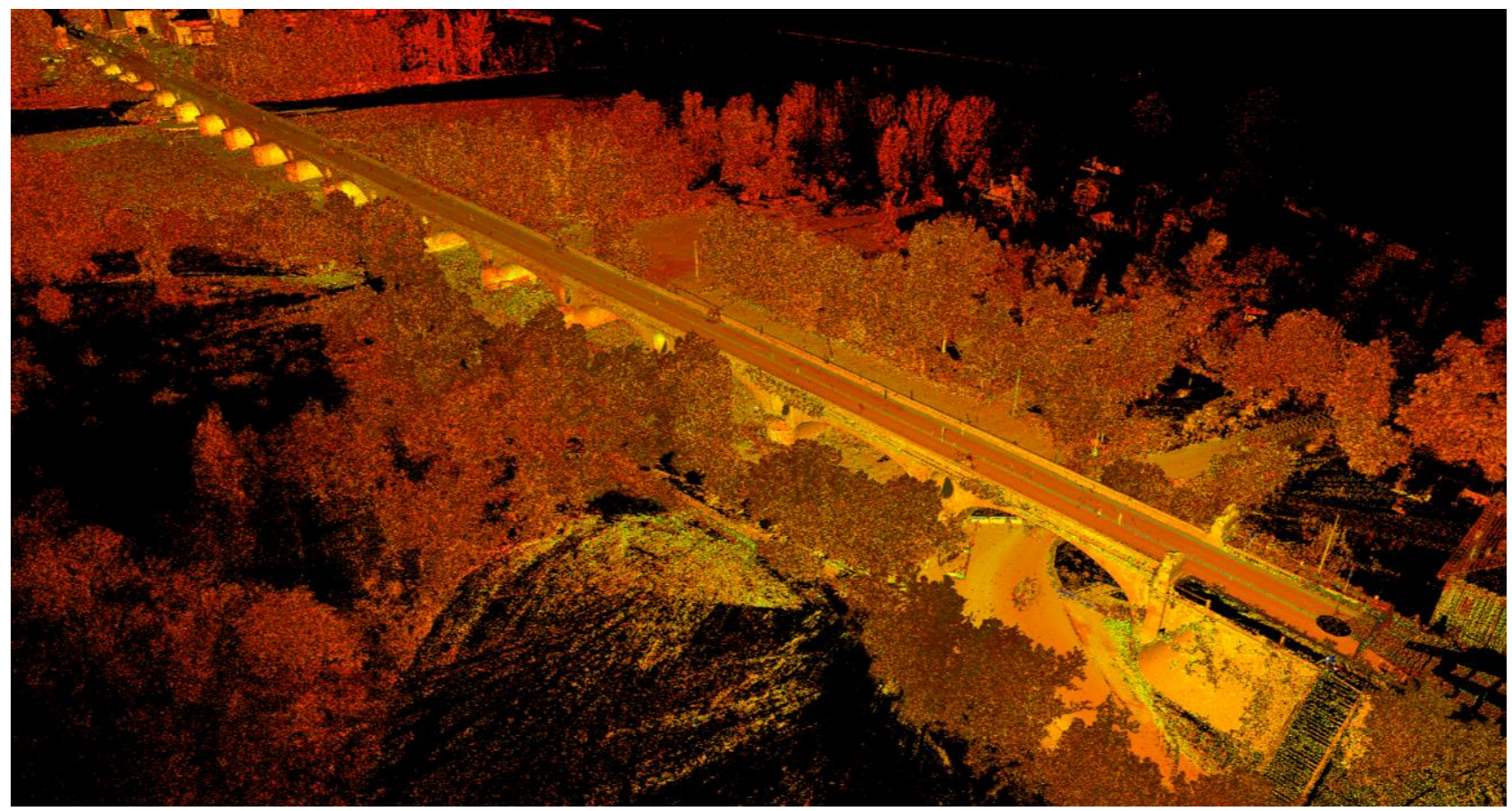

Figure 2. Pont cloud obtained by laser-scanner survey.

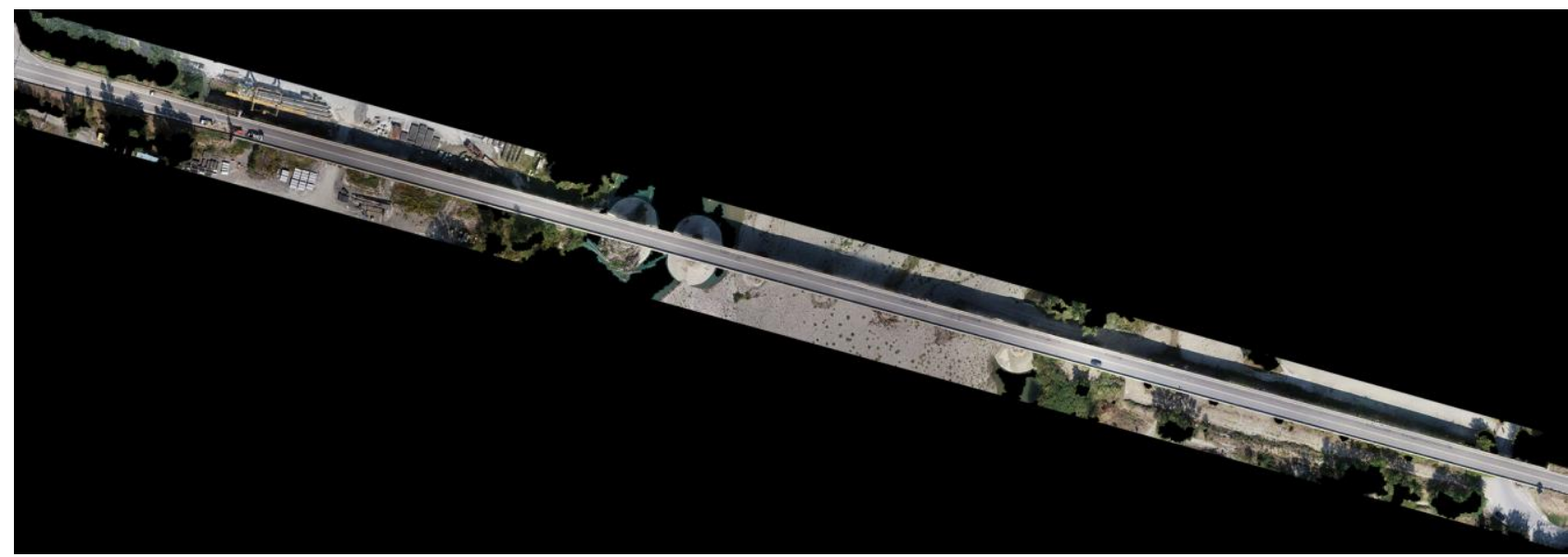

Figure 3. Orthophoto of survey area. 


\section{ANALYZING THE GEOMETRICAL SURVEY RESULTS TO INSPECT STRUCTURAL DEFORMATIONS}

\subsection{The historical monitoring method}

The pieces of information that can be obtained through a specific interpretation of the geometrical survey data represent powerful tools for the comprehension of the present structural conditions of the bridge and precious elements for the validation of any numerical model. Identifying and quantifying soil settlements and deformations allow to define boundary conditions that retrace the history of the monument. A structural analysis that disregards these data can instead lead to completely unreliable results (Bruno et al., 2017, Coïsson et al., 2018)

Thanks to the high precision survey previously described, it has been possible to compare the realized infrastructure and the original project and to inspect the geometrical unevenness. Indeed, the measured differences between them can give important information on the historical transformations of the bridge during the construction phase, and on the deformation suffered by the structure during time, thus constituting the "historical monitoring" method (Coïsson and Ottoni, 2015).

\subsection{Main results}

The analysis of the geometrical survey allowed to compare the levels of the pillars and of the keystones of the 20 arches. Under the hypothesis that none of these elements could increase its own level in time and that the original geometry had pillars and keystones all at the same level, the difference between the element (pillar and keystone) with the maximum height and the others was measured and reported on a graph (in Figure 4 the detail of the arch n. 2, starting from the West side), depicting enhanced distances: in blue the measures referred to the pillars, in orange the ones referred to the keystones. All the measures are also reported in Table 1, together with the measures of the differences between the designed arch rise and the surveyed one.

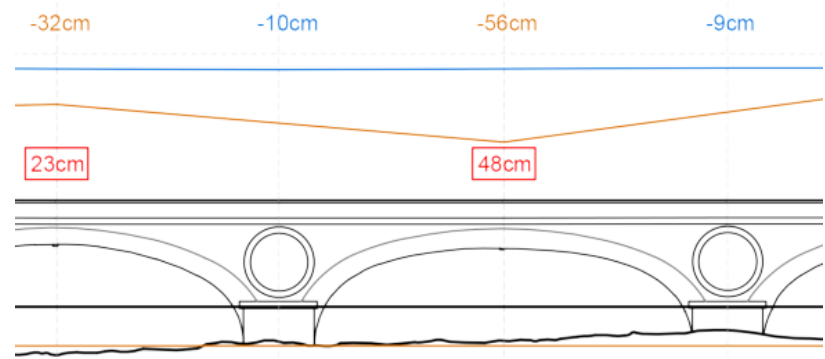

Figure 4. The differences in the piles and keystones levels (here reported only for the arch n. 2) supply indications about the possible settlements and deformations suffered by the structure.

The first observation that can be made is that the pillars show quite limited settlements, not larger than $10 \mathrm{~cm}$ on the entire $600 \mathrm{~m}$ of length and not larger than $6 \mathrm{~cm}$ between two consequent pillars. Heavy underpinning interventions have been carried out in the last decades of the $20^{\text {th }}$ century on the foundations of the central pillars, but more recent tests have shown that under the added reinforced concrete slab, large voids are present due to the river undermining action. The surveyed data seem to indicate that probably the past interventions on the foundations of pillars 5 to 14 (from the
West end) have been effective: indeed, it can be noticed that the pillars in the middle have lower settlements than the ones at the extremities: the mean differential settlement is just $2.9 \mathrm{~cm}$ in the strengthened pillars, while it is $9 \mathrm{~cm}$ in the first 5 pillars from the West end, $5.4 \mathrm{~cm}$ in the first 5 pillars from the East end. Therefore, the soil settlements do not seem to represent currently a serious issue. As a consequence, it is difficult to explain the reasons of the much larger differences in the keystone levels, and the consequent differences in the arches rises, that reach $56 \mathrm{~cm}$ in the second arch (starting from the West side) and are larger than $20 \mathrm{~cm}$ in the majority of the arches. This can be ascribed both to defects in the building phase (which could originally differ from a geometrically perfect shape) and/or to settlements and deformations in the two centuries long life of the structure, with its increased loads and numerous damaging events (floods, car accidents, bombings, earthquakes).

\begin{tabular}{|c|c|c|c|}
\hline Arch number & $\begin{array}{r}\text { Diffel } \\
\text { the hig }\end{array}$ & $\begin{array}{l}\text { n level from } \\
\text { orresponding } \\
\text { ht }[\mathrm{cm}]\end{array}$ & $\begin{array}{l}\text { Difference in the } \\
\text { arch rise }\end{array}$ \\
\hline WEST & $\begin{array}{c}\text { Pillar } \\
-9\end{array}$ & Keystone & \\
\hline 1 & & -18 & -9 \\
\hline 2 & -10 & -56 & -48 \\
\hline 3 & -9 & -32 & -23 \\
\hline 4 & -8 & -38 & -29 \\
\hline 5 & -2 & -20 & -15 \\
\hline 6 & -4 & -12 & -9 \\
\hline 7 & -1 & -22 & -19 \\
\hline 8 & -4 & -35 & -32 \\
\hline 9 & -3 & -35 & -31 \\
\hline 10 & -3 & -24 & -21 \\
\hline 11 & 0 & -21 & -19 \\
\hline 12 & -3 & -37 & -35 \\
\hline 13 & -4 & -15 & -12 \\
\hline 14 & -3 & -13 & -10 \\
\hline 15 & -5 & -24 & -20 \\
\hline 16 & -5 & -10 & -5 \\
\hline 17 & -2 & -6 & -3 \\
\hline 18 & -4 & -13 & -10 \\
\hline 19 & -8 & 0 & -6 \\
\hline $\begin{array}{l}20 \\
\text { EAST }\end{array}$ & -8 & -6 & -2 \\
\hline
\end{tabular}

Table 1. Settlements of piers and keystones and differences in the arches drops, measured on the geometrical survey. 
Also, the evolution of the deformation might have been uneven in time: historical documents (Cocconcelli, 1825) refer about problems occurred during the removal of the centering in some arches, but further information is needed for a complete frame of the ancient structural disorders, that could allow to define more precisely the settlements evolution of each pillar and each arch.

Differently from the pillar settlements, the arch deformations are lower on the East part, towards Parma, and gradually increase towards the West end, although with several irregularities. A specific inspection to understand the reasons of the non-uniformity of these deformations was then carried out. Some damages were found in the Western arches, with the collapse of a portion of the external leave of an arch and a crack pattern on the front which could be connected to a differential soil settlement between pillars 4 and 5. Moreover, a comparison between the original profile of the arches and the surveyed one pointed out in some cases the loss of symmetry, connected to a damage mechanism which influence the global behaviour.

\section{THE PHOTOGRAMMETRIC MODEL AS AN INSTRUMENT FOR THE CONSERVATION PROJECT}

\subsection{Methods}

The Maria Luigia bridge presents various types of decay, unevenly diffused on its masonry surfaces. As a matter of fact, this structure is constantly subject to highly-erosive external actions (pollution, weathering, river overflows, freezing and thawing cycles, thermal expansion, infiltration of salts from the road, etc.). Periodically, restoration works are needed with the aim to ensure the preservation of the monument, even if it is known that some degradations will appear again in the future, because they are caused by external solicitation that cannot be removed.

In order to plan a strategy of intervention and maintenance, with a good balance of the available resources, a survey of the decay phenomena that fully represents the real conditions of the bridge is the first step to be carried out. In this case, it was particularly important to pay attention not only to the correct identification of the degradation but also to the correct evaluation of the extent of the pathology. In consideration of the high cost required for restoration (about 150 euros per square meter, as a mean value) and, in particular, in consideration of the huge size of the structure (surface area about 11.700 square meter), underestimating or overestimating the value of the areas which need an intervention, would lead to significant errors in the evaluation of costs.

The mapping of the material decay of the Maria Luigia bridge started from in-situ inspections of the masonry surfaces in order to achieve a correct identification of the deterioration types and levels. Then, the decay patterns have been represented on textured front elevations and plan views provided by the laser scanner and photogrammetric surveys.

While the representation of the decays on the fronts reproduces the state of conservation with minimal geometrical approximations of the areas which need interventions, the representation of the decays on the arches involved more difficulties. The spans of the bridge are made of three-centered depressed arches and, moreover, each arch has deformations and geometric irregularities.

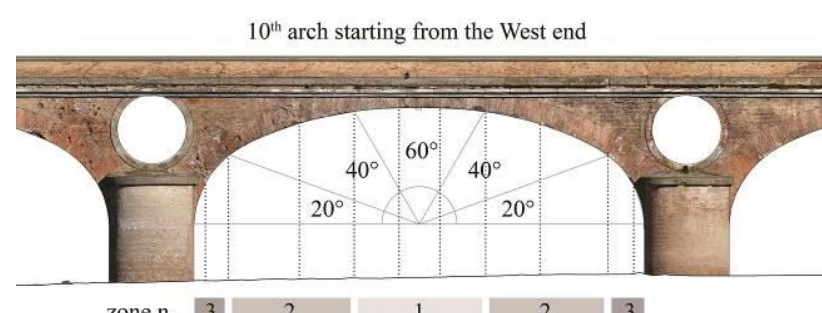

zone $\mathrm{n}$.
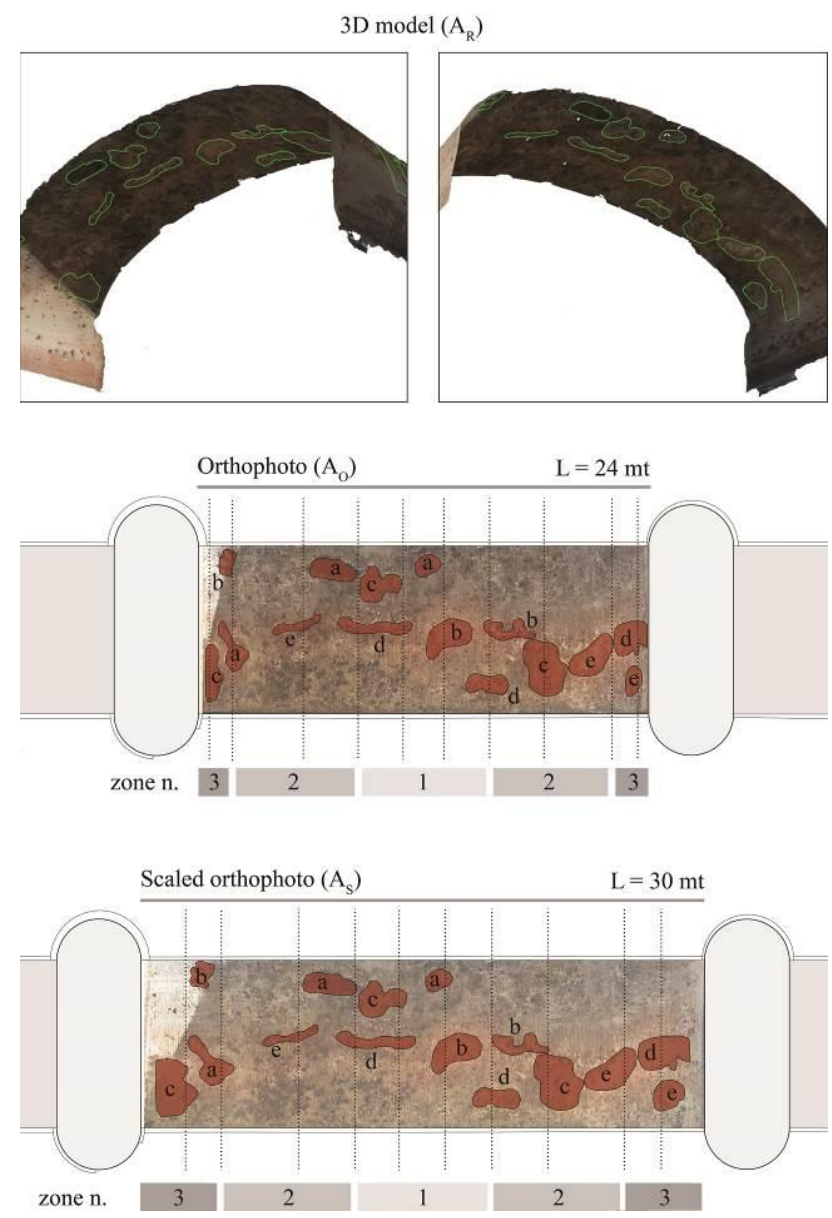

Figure 5. Mapping of decay on the $10^{\text {th }}$ arch (starting from the West end of the bridge) and measurement of fifteen areas through three different methods (using a 3D model, an orthophoto and a scaled orthophoto).

In order to obtain a flat projection of the three-centered arch texture, as a realistic base for the decay mapping, a photogrammetric survey have been carried out as described in the previous sections. The use of a textured 3D model for decay documentation was considered as the most accurate (Chiabrando et al., 2017, Tsilimantou et al., 2016), although the amount of data needed for this procedure would have been hardly manageable, requiring machines with high computational performance and considerable resources of time. Alternative methods have therefore been investigated in order to reach a good compromise between ease and speed of application and approximation in the quantification of the areas of deteriorated surfaces (D'Agostino, 2010). Another option, in fact, is represented by the use of an intrados orthophoto which represents a 2D orthographic projection of the information acquired by the (oriented) images of the photogrammetric image block. However, at the time being, commercial software allows 
only to consider planar (or in some cases cylindrical) projection surfaces for orthophoto generation. In both cases (planar or cylindrical projection) the three-centered depressed arch orthophoto would show significant deformations, and consequently would lead to significant errors in area quantifications, in particular for the areas near the pillars. The projection on a cylinder would increase the precision, but even in this case the full correspondence with the real surfaces is not guaranteed since the three different curvatures of the masonry arch should be approximated in a constant single curvature surface.

Another method was therefore considered. Since the actual surface of the vault can still be considered as an approximate varying single-curvature surface, the planar (or cylindrical) projection orthophoto obtained with the previous method can be re-scaled pixelwise along the bridge longitudinal direction, considering the angle between the mean longitudinal section of each single arch and the orthophoto projection surface.

As an example, the decay located in the tenth arch (starting from the West end) has been considered and, in the specific, four pathologies (erosion, deposit, salt crust and salt efflorescence) that are distributed among all the surface, form the springers to the keystone. Three different methods have been used to measure the areas of these decays and then the values have been compared in order to identify the best optimization in the evaluation of the real extent of the degradations.

The arch has been divided into three zones, according to the curvature of its profile, as shown in Figure 5: the first zone concerns the central part of the arch (arch of a sector with a central angle of $60^{\circ}$ ) while the second zone concerns the haunches (arch of a sector with a central angle of $40^{\circ}$ ) and the third zone concerns the springers (arch of a sector with a central angle of $20^{\circ}$ ). For each zone, five decays, identified with a letter from "a" to "e", have been mapped and measured.

\subsection{Main results}

The first method measures this area by recreating the decay surface on the 3D model provided by the photogrammetric and laser scanner survey. In particular, the perimeters of the degradations have been identified directly on the textured mesh model and, then, the discretized surfaces have been reconstructed starting from these curves. This method allows to obtain a realistic and direct value of the areas of intervention $\left(A_{R}\right)$ and, consequently, it leads to a correct evaluation of costs. However, the currently available tools do not allow to easily carry out this operation because of difficulties in recreating the surfaces and the high computing power required to manage such big data.

The second method consisted in using a flat (planar) projected orthophoto of the intrados. The accuracy of the areas projected on the horizontal plane $\left(\mathrm{A}_{\mathrm{O}}\right)$ varies differently according to the slope of the surface w.r.t. the horizontal projection plane: in the central part the percent difference between $A_{R}$ and $A_{O}$ is equal to $4 \%$, while it increases in the second zone $(9 \%)$ and especially in the third zone, where it reaches $40 \%$ (as shown in table 2). These values underline how the geometrical deformations in the projection implies significant errors in the evaluation of the extent of decay, especially near the springers. Given that this flat texture presents deformations related to the angle between the curved surface and the projection plane, the values should be corrected with a scale factor, related to that angle. Anyway, the application of this method would be excessively time consuming.

The third method consisted in a differential scaling along longitudinal sections of each single arch of the planar orthophoto considered in the previous method. Even in this case, the accuracy of the areas identified on this flat texture $\left(A_{S}\right)$ varies differently on the surface but the percent difference between $A_{R}$ and $A_{S}$ is smaller than in the second case. In the specific, it is equal to $3 \%$ in the first zone, $4 \%$ in the second one and $6 \%$ in the third one (as shown in table 2). This result shows a reasonable correspondence between the real extent of degradation and its representation through this method, with an oversizing of less than $10 \%$. Note that the error near the springers has considerably reduced, allowing the mapping of decay directly on this representation also in the third zone. On the other hand, the quality of the texture is not high but this does not influence the evaluation of the extent of degradations.

\begin{tabular}{|c|c|c|c|c|c|}
\hline \multirow[b]{2}{*}{ Decay } & \multirow{2}{*}{$\begin{array}{c}\begin{array}{c}\mathbf{1}^{\text {st }} \\
\text { Meth }\end{array} \\
\mathbf{A}_{\mathbf{R}} \\
\left(\mathrm{m}^{2}\right)\end{array}$} & \multicolumn{2}{|c|}{$2^{\text {nd }}$ Meth } & \multicolumn{2}{|c|}{$3^{\text {rd }}$ Meth } \\
\hline & & $\begin{array}{c}\mathbf{A}_{\mathbf{o}} \\
\left(\mathrm{m}^{2}\right)\end{array}$ & $\begin{array}{c}\mathbf{A}_{\mathbf{R}}-\mathbf{A}_{\mathbf{O}} \\
(\%)\end{array}$ & $\begin{array}{c}\mathbf{A}_{\mathbf{S}} \\
\left(\mathrm{m}^{2}\right)\end{array}$ & $\begin{array}{c}\mathbf{A}_{\mathbf{R}}-\mathbf{A}_{\mathbf{S}} \\
(\%)\end{array}$ \\
\hline \multicolumn{6}{|c|}{$1^{\text {st }}$ ZONE } \\
\hline $1 \mathrm{a}$ & 1,23 & 1,23 & 1 & 1,23 & 1 \\
\hline $1 b$ & 3,41 & 3.36 & 1 & 3,46 & -1 \\
\hline $1 \mathrm{c}$ & 2,87 & 2,79 & 3 & 2,92 & -2 \\
\hline $1 \mathrm{~d}$ & 2,04 & 2,00 & 2 & 2,07 & -2 \\
\hline \multirow[t]{2}{*}{$1 \mathrm{e}$} & 2,65 & 2,26 & 15 & 2,40 & 9 \\
\hline & & & average 4 & & average 3 \\
\hline \multicolumn{6}{|c|}{$2^{\text {nd }}$ ZONE } \\
\hline $2 a$ & 2,40 & 2,31 & 3 & 2,51 & -5 \\
\hline $2 b$ & 1,91 & 1,86 & 3 & 1,98 & -3 \\
\hline $2 \mathrm{c}$ & 5,53 & 5,23 & 4 & 5,65 & -2 \\
\hline $2 \mathrm{~d}$ & 1,33 & 1,06 & 20 & 1,21 & 9 \\
\hline \multirow[t]{2}{*}{$2 \mathrm{e}$} & 3,77 & 3,28 & 13 & 3,79 & -0 \\
\hline & & & average 9 & & average 4 \\
\hline \multicolumn{6}{|c|}{$3^{\text {rd }}$ ZONE } \\
\hline $3 a$ & 2,39 & 1,84 & 23 & 2,54 & -6 \\
\hline $3 b$ & 1,24 & 0,92 & 26 & 1,32 & -6 \\
\hline $3 \mathrm{c}$ & 3,84 & 2,07 & 46 & 4,06 & -6 \\
\hline $3 d$ & 6,21 & 2,72 & 56 & 5,96 & 4 \\
\hline \multirow[t]{2}{*}{$3 \mathrm{e}$} & 2,20 & 1,15 & 48 & 2,06 & 6 \\
\hline & & & average 40 & & average 6 \\
\hline
\end{tabular}

Table 2. Values of areas of decay measured through a 3D model $\left(A_{R}\right)$, an orthophoto $\left(A_{O}\right)$ and a scaled orthophoto $\left(A_{S}\right)$ and comparison between $A_{O}$ and $A_{R}$ and between $A_{S}$ and $A_{R}$

Therefore, waiting for a future development of a direct method for tridimensional mapping, which could provide the most realistic data, it has been shown how the last method proposed could implement the commercial software in order to help the user to easily represent an irregular curved texture on a flat plane with a good ratio between computing time and quality of the correspondence to the real area extent.

\section{CONCLUSIONS}

In conclusion, the present work highlights the fundamental role of an interdisciplinary investigation when historical infrastructures are concerned: the structural analysis has to be based not only on the present day survey, but also on its 
interpretation in terms of deformations, on the indications of the ancient treaties and on their comparison with the present state. Both the geometrical and photogrammetric surveys have supplied useful information for the identification of structural problems and for the quantification of material decay phenomena, as a base for future interventions. An integrated approach that can only be carried out with a multidisciplinary group, with the common aim of understanding the monument and working for its preservation.

\section{ACKNOWLEDGEMENTS}

The authors gratefully acknowledge the contribution of the Parma Municipality to the development of this research and the helpfulness of Eng. Marcello Bianchini Frassinelli in the coordination of the related works.

\section{REFERENCES}

Chiabrando, F., Lo Turco, M., Rinaudo, F., 2017. Modeling the decay in an HBIM starting from 3D point clues. A followed approach for cultural heritage knowledge, Proceedings of International Archives of the Photogrammetry, Remote Sensing \& Spatial Information Sciences 42.

Tsilimantou, E., Delegou, E., Ioannidis, C., Moropoulou, A., 2016. Geoinformation techniques for the $3 \mathrm{D}$ visualisation of historic buildings pathology and representation of a building's pathology, Proceedings of Fourth International Conference on Remote Sensing and Geoinformation of the Environment (RSCy2016) 9688, 968810.

D'Agostino, D., 2010. A methodology of damage assessment in cultural heritage: Graphic decay mapping, Proceedings of ICAMS-3rd International Conference on Advanced Materials and Systems, Bucharest, 433-438.

Bruno, N., Coïsson, E., and Cotti, M., 2017. Laser-scanner survey of structural disorders: an instrument to inspect the history of Parma cathedral's central nave. International Archives of the Photogrammetry, Remote Sensing \& Spatial Information Sciences, 42.

Coïsson, E., Cotti, M., \& Ottoni, F., 2018. Deformation measurement as a calibration tool for structural modelling of built heritage. In IOP Conference Series: Materials Science and Engineering (Vol. 364, No. 1, p. 012086). IOP Publishing.

Cocconcelli, A.,1825. Descrizione dei progetti e lavori per l'innalzamento dei due ponti sul Taro e sulla Trebbia, Parma (in Italian).

Basteri, M.C., Dall'Acqua, M., 2004. Unire sponde: il Ponte sul Taro dall'età Romana ad oggi, Parma, Tecnostampa Edizioni (in Italian).

Benvenuto, E., 1981. La Scienza delle Costruzioni nel suo sviluppo storico, Sansoni, Firenze (in Italian).

Coïsson, E., and Ottoni, F., 2015. Structural Monitoring of Historical Constructions: Increasing Knowledge to Minimize Interventions. In Built Heritage: Monitoring Conservation Management (pp. 83-92). Springer, Cham. 